\title{
No need for panic about AIDS
}

\section{Acquired immune deficiency disease, now frequent among male homosexuals in the United States, is not this century's Black Death. The most urgent need is to understand what is going on.}

THERE is now a serious danger that alarm about the disease physicians call acquired immune deficiency syndrome (unhelpfully, AIDS for short) will get out of hand. For the characteristics of this previously unrecognized and perhaps non-existent condition are so alarming that the temptation to portray it as a disease invited by a decadent civilization - a kind of latter-day version of the fate of Sodom and Gomorrah - is almost irresistible. Television companies are in danger of succumbing like flies to the temptation, for which reason it is important that the nature of the social problem that has arisen should be quantitatively as well as qualitatively understood.

Briefly, the condition is disturbing because it is either new or newly recognized, and because the mortality among those affected is high, probably more than 70 per cent. But the numbers of people among whom the disease has been recognized in the past few years is of the order of a thousand, most of whom are male homosexuals. Although successive waves of the mediaeval plague took several decades to work their way around the world, the communities in which infection became established would have been in no doubt (if they had known about the germ theory of disease) that some novel infectious agent had reached them.

The occurrence of acquired immune deficiency is, by comparison, covert. The first reports that something novel was afoot came in June 1981, and were based on the observation by the Center for Disease Control at Atlanta, Georgia, that five male homosexuals in Los Angeles had been diagnosed in the previous eighteen months as suffering from pneumonia caused by Pneumocystis carinii, a protozoan infection rarely seen by clinicians except in patients receiving immunosuppressive therapy. With the benefit of hindsight, the Atlanta centre was able to find further cases of this rarely seen pneumonia and also a geographically related cluster of patients who sufferered or had died from Kaposi's sarcoma, a rare skin malignancy recognized at least five years ago as an accompaniment of immunosuppressive treatment (see Gange, R.W. and Jones, E.W., Clin. exp, Dermatol. 3, 135; 1978). The hunt for acquired immune deficiency disease, and for an explanation of it, has been on since then.

At this still early stage, it is too soon to know the scale of the problem, or even how long it has been with us. Even if the causative agent is some transmissible agent, the fact that those who contract the disease turn up with some well-known but unrelated infection is bound to complicate diagnosis, which implies that the incidence of the disease is probably even now underestimated. Equally, however, since physicians on the look-out for a newly reported condition are less likely than in previous years to overlook the significance of an unusual condition, there is no easy way of telling how many people acquired immune deficiency before Atlanta recognized the importance of the first five cases reported from Los Angeles.

Indeed, the experience of the past two years, since the first reports in June 1981, suggests that there are many more than 1,000 cases to be identified. Among those so far found to be affected, male homosexuals predominate but acquired immune deficiency has also been found among women whose sexual partners are male homosexuals. Moreover, there are at least two cases in which newborn children have acquired the condition, one apparently in utero and the other as a result of blood transfusion. It is now recognized that the disease is unusually uncommon among those taking drugs intravenously, among their sexual partners, among those (such as haemophiliacs) dependent on blood products and - most surprisingly among Haitian immigrants to the United States without any of the predisposing dependencies of those among the indigenous population who succumb. But, so far as is known, the results of a study of immune deficiency in Haiti have not yet been reported.

Even the notion that immune deficiency is acquired by means of a transmissible agent, a virus for example, is open to dispute, or at least unproven. The evidence is only circumstantial, while the recent report (Ewing, E.P. et al., New Eng. J. Med., 7 April) of the presence of structures called vesicular rosettes in electron micrographs of the cytoplasm of cells from affected patients but only rarely in that from the cells of normal people is inconclusive, for the rosettes resemble no known virus. On balance, however, the epidemiology of the disease, and the observation that those dependent on blood products are especially at risk, suggests that something carried in the blood of affected people must be the transmitting agent. The peculiar susceptibility of Haitian immigrants to the United States who are apparently neither homo- sexuals nor drug takers suggests some genetic predisposition.

When everybody is talking about parts of the human genome that may be mobilized to form viruses, it is natural that people's imaginations should take in the notion that integrable viruses such as human T-cell leukaemia virus (see Nature, 14 April) may be involved. But the presence of antibodies against such a virus may simply be a consequence of the impaired cell-mediated immunity of those with acquired immune deficiency. As it happens, male homosexuals not yet stricken with acquired immune disease tend also to carry antibodies against other viruses, hepatitisB virus, for example, but that, too, is an inconclusive observation. Even the link between cytomegalovirus and Kaposi's sarcoma is inconclusive for the same reasons

What, in the circumstances, should be done? Finding the cause of the disease is the most urgent need but, fortunately, the techniques are now available. The hope of treating those with acquired immune deficiency must be a matter of chance - an interferon or some other lymphokine may work, but only by sheer luck. Even the observation that, in many patients with acquired immune deficiency, the ratio of "helper" to "suppressor" T-cells is decreased is of no help when so little is known of the functions of these different types of cells in real life, or of their genetic origins. But mercifully, the disease whatever its causation - is neither especially infectious (sexual contact seems necessary) nor certain in its effects. And the incubation period of a virus must be measured in months, not days. So there is a chance that the disease, whatever it is, will be understood before it has become too widely established, as bubonic plague once was.

Meanwhile, and for strictly prophylactic purposes, male homosexuals should be persuaded to change their ways. By all accounts, fears of acquired immune deficiency have already had a profound effect in cities where homosexuality is frequent. The pathetic promiscuity of male homosexuals is the most obvious threat to public health, but is probably no more serious now than it was before homosexuality ceased to be illegal. The use of substances such as amyl nitrite to assist the mechanics of male homosexuality, but which appear by accident to be immunosuppressants as well, should be discouraged. The fear of AIDS will no doubt help. 